\title{
Development of a Prognostic Model to Predict Mortality after Traumatic Brain Injury in Intensive Care Setting in a Developing Country
}

\author{
Mini Jayan ${ }^{1}$ Dhaval Shukla1,2,๑ Bhagavatula Indira Devi ${ }^{1,2}$ Dhananjaya I. Bhat ${ }^{3}$ Subhas K. Konar ${ }^{1}$
}

${ }^{1}$ Department of Neurosurgery, National Institute of Mental Health and Neurosciences, Bengaluru, India

${ }^{2}$ NIHR Global Health Research Group on Neurotrauma, University of Cambridge, Cambridge, United Kingdom

${ }^{3}$ Department of Neurosurgery, RV Aster Hospital, Bengaluru, India
Address for correspondence Bhagavatula Indira Devi, MCh, Department of Neurosurgery, National Institute of Mental Health and Neurosciences, Bengaluru 560029, Karnataka, India (e-mail: bidevidr@gmail.com).

J Neurosci Rural Pract 2021;12:368-375.

\begin{abstract}
Keywords

- traumatic brain injury

- outcome prediction

- intensive care unit

Objectives We aimed to develop a prognostic model for the prediction of in-hospital mortality in patients with traumatic brain injury (TBI) admitted to the neurosurgery intensive care unit (ICU) of our institute.

Materials and Methods The clinical and computed tomography scan data of consecutive patients admitted after a diagnosis TBI in ICU were reviewed. Construction of the model was done by using all the variables of Corticosteroid Randomization after Significant Head Injury and International Mission on Prognosis and Analysis of Clinical Trials in TBI models. The endpoint was in-hospital mortality.

Results A total of 243 patients with TBI were admitted to ICU during the study period. The in-hospital mortality was $15.3 \%$. On multivariate analysis, the Glasgow coma scale (GCS) at admission, hypoxia, hypotension, and obliteration of the third ventricle/basal cisterns were significantly associated with mortality. Patients with hypoxia had eight times, with hypotensions 22 times, and with obliteration of the third ventricle/basal cisterns three times more chance of death. The TBI score was developed as a sum of individual points assigned as follows: GCS score 3 to 4 (+2 points), 5 to $12(+1)$, hypoxia $(+1)$, hypotension $(+1)$, and obliteration third ventricle/basal cistern $(+1)$. The mortality was $0 \%$ for a score of " 0 " and $85 \%$ for a score of " 4 ."

Conclusion The outcome of patients treated in ICU was based on common admission variables. A simple clinical grading score allows risk stratification of patients with TBI admitted in ICU.
\end{abstract}

\section{Introduction}

A prognostic model is a statistical model, or a mathematical equation, that includes two or more prognostic factors to calculate the probability of a predefined outcome. ${ }^{1}$ In medical research, the outcome is often dichotomized, for

published online April 1, 2021
DOI https://doi.org/

$10.1055 / \mathrm{s}-0041-1726623$ ISSN 0976-3147 example, alive or dead. Prognostic models predicting outcomes from initial clinical severity, radiological findings, and laboratory values can provide prognostic information for patients' families, help in early clinical decision-making, and patient stratification and selection in clinical studies.,3 A useful model should be clinically and methodologically 
valid. More than 100 prognostic models have been described for traumatic brain injury (TBI) but have methodological issues. Most of these models are based on small sample sizes. The statistical methods used for the development of these models are not robust. More than $90 \%$ of these models come from high-income countries, hence not useful in resource-limited settings. Only one-fifth of these models are user-friendly. ${ }^{14-6} \mathrm{An}$ important attribute of a model is external validation.

External validation aims to assess the performance of a prognostic model in a different but plausibly related population. External validation is essential to support the generalizability of prognostic models and to provide evidence that the model does, in fact, accurately predict outcomes. Only one-tenth of the models for prognostication of TBI have been externally validated. ${ }^{4}$ Among these prognostic models, the Corticosteroid Randomization After Significant Head Injury (CRASH) trial and International Mission on Prognosis and Analysis of Clinical Trials in TBI (IMPACT) database and are constructed with sophisticated statistical methodology, large datasets, and have been externally validated. ${ }^{7-11}$ The CRASH model predicts 2 weeks of mortality and 6 months of an unfavorable outcome. This model is based on admission characteristics of patients and has not been validated in the intensive care unit (ICU) setting in developing countries. We aimed to develop a prognostic model incorporating clinical and radiological variables for the prediction of in-hospital mortality in moderate-to-severe TBI using variables from the CRASH and IMPACT model.

\section{Materials and Methods}

\section{Patients}

This study was done at the National Institute of Mental Health and Neurosciences, a tertiary-level referral center for treating neurological, neurosurgical, and psychiatric patients. It has a separate facility for trauma cases that serves patients from Bengaluru and neighboring districts. Patients with TBI, who have a different registration process, are evaluated by neurosurgery residents. Their data are entered in a structured "head injury proforma," which consists of comprehensive clinical and computed tomographic (CT) scan findings. A qualified neurosurgeon on duty verifies these data. Data of all consecutive patients admitted to our ICU for the treatment of moderate-to-severe TBI during 11 months period from January 2012 to November 2012 were analyzed retrospectively. The inclusion criteria were a history of isolated head injury and Glasgow coma scale (GCS) 3 to 12 at the time of admission to ICU. All patients were managed with standard principles of management of TBI. Intracranial pressure monitoring or any other invasive neuromonitoring was not done for any patients. Patients of all age groups and both genders were included. Patients with penetrating brain injury and significant extracranial injury (defined as an injury requiring admission in its own right) were excluded. The intensive care management of these patients was performed as per the Brain Trauma Foundation guidelines except for intracranial pressure monitoring. The sample size was calculated by using OpenEpi, utilizing the data from the CRASH study. ${ }^{12}$ The CRASH study included 7,526 patients from low- to middle-income countries with mortality of $20.7 \%$. For expected mortality of $20.7 \% \pm 5 \%$, and $95 \%$ confidence level, the estimated sample size was 245 . As this is a retrospective observational study and can be considered a clinical audit rather than research; hence, the institute ethics committee approval was not taken.

\section{Prognostic Variables}

We recorded all the clinical and radiological variables mentioned in the CRASH and IMPACT model. The following clinical variables were recorded: age, GCS, and pupillary reaction. The following CT scans variables were recorded: petechial hemorrhages $(<5 \mathrm{~mm}$ specks of bleed seen in the location of lesions of diffuse axonal injury lesion), obliteration of third ventricle/basal cisterns, subarachnoid hemorrhage, midline shift, and nonevacuated hematoma. Besides, secondary complications were also recorded. These included hypotension (systolic blood pressure $<90 \mathrm{~mm} \mathrm{Hg}$ ), hypoxia $\left(\mathrm{pO}_{2}<60 \mathrm{~mm} \mathrm{Hg}\right.$ ), and hyperthermia (temperature $>37.5^{\circ} \mathrm{C}$ ). We used in-hospital mortality as the outcome of interest.

\section{Model Construction and Performance}

Data of patients were used for model construction. All data were entered and analyzed in SPSS ver. 20 (IBM, Armonk, New York, United States). Mann-Whitney U test was used for nonparametric continuous univariate analysis, and the Chi-square test or Fisher exact test was used for categorical univariate analysis. A series of univariate logistic regression was performed to identify the factors associated with the survival of patients. The variables that were statistically significant in the univariate analysis were included in the multivariable analysis. This was done after taking care of the assumptions of multivariable logistic regression analysis including multicollinearity. Since GCS at admission and GCS at discharge showed a correlation, only the former variable was included in the analysis. Since the percentage of missingness was high in the variable "platelet count," it was excluded from the multivariable analysis.

Discrimination is the model's ability to separate between those who die and survive. The discriminative power (predictive power) of the model was assessed by calculating the area under the receiver operating characteristics (ROC) curve (AUC). A model with AUC of 0.90 to 1 is said to have excellent discrimination power.

\section{Results}

Data of 243 patients were analyzed. The age range was from 1 to 85 years. The male to female ratio was 5.5:1. The mean ICU stay was $8.3 \pm 7.2$ days. The in-hospital mortality was $15.3 \%$. The average time to death was $7.5 \pm 4.6$ days. The GCS at admission, motor score at admission, intubation at admission, pupillary reaction at admission, hypoxia, hypotension, obliteration third ventricle/basal cistern, platelets count, and GCS at discharge were the factors identified as statistically significant (- Table $\mathbf{1}$ ). 
On multivariate analysis, GCS at admission, hypoxia, hypotension, and obliteration of the third ventricle/basal cisterns were significantly associated with mortality (-Table 2 ). The odds of survival were $15.5 \%$ more for one unit increase in GCS at the time of admission. Patients with hypoxia had eight times (95\% confidence interval: 2.187-30.481; $p=0.025$ ) more chance of death compared with those who did not have hypoxia. The odds of death were nearly 22 times more for

Table 1 Comparison between dead and alive groups $(n=243)$

\begin{tabular}{|c|c|c|c|}
\hline Variable & Dead $(n=41)$ & Alive $(n=202)$ & $p$-Value \\
\hline \multicolumn{4}{|l|}{ Demography } \\
\hline \multicolumn{4}{|l|}{ Gender } \\
\hline Male & 34 (83\%) & 171 (85\%) & 0.728 \\
\hline Female & $7(17 \%)$ & $31(15 \%)$ & \\
\hline Age $(y)^{a}$ & $38.7 \pm 15.0$ & $38.4 \pm 17.9$ & 0.91 \\
\hline Children ( $\leq 16$ y) & $2(4.9 \%)$ & $22(10.8 \%)$ & 0.38 \\
\hline \multicolumn{4}{|l|}{ Injury characteristics } \\
\hline Place of injury & & & 0.051 \\
\hline Urban & $33(80 \%)$ & $131(65 \%)$ & \\
\hline Rural & $8(20 \%)$ & $71(35 \%)$ & \\
\hline Cause of injury & & & 0.13 \\
\hline Road traffic accident & $24(59 \%)$ & $142(70 \%)$ & \\
\hline Fall & $9(22 \%)$ & $41(20 \%)$ & \\
\hline Assault & $1(2 \%)$ & $3(2 \%)$ & \\
\hline Animal related injury & $0(0 \%)$ & $4(2 \%)$ & \\
\hline Others & $7(17 \%)$ & $12(6 \%)$ & \\
\hline Extracranial injury & $6(15 \%)$ & $36(18 \%)$ & 0.62 \\
\hline \multicolumn{4}{|l|}{ Symptoms at admission } \\
\hline Vomiting & $14(34 \%)$ & 95 (47\%) & 0.13 \\
\hline Loss of consciousness & $40(98 \%)$ & $176(87 \%)$ & 0.053 \\
\hline Seizures & $3(7 \%)$ & $9(4 \%)$ & 0.44 \\
\hline ENT bleed & $18(44 \%)$ & $84(42 \%)$ & 0.78 \\
\hline \multicolumn{4}{|c|}{ Physiological derangements at admission } \\
\hline GCS & $6.5 \pm 2.6$ & $8.4 \pm 3.1$ & $<0.001$ \\
\hline Severe TBI $(\mathrm{GCS}<9)$ & $33(80.5 \%)$ & $102(50.3 \%)$ & $<0.001$ \\
\hline Motor score & $3.6 \pm 1.4$ & $4.5 \pm 1.3$ & 0.001 \\
\hline Pupillary reaction & & & 0.028 \\
\hline Bilateral reacting & $9(22 \%)$ & $93(46 \%)$ & \\
\hline Unilateral dilatation & $7(17 \%)$ & $39(19 \%)$ & \\
\hline Bilateral dilatation & $22(54 \%)$ & $61(30 \%)$ & \\
\hline Unable to assess & $3(7 \%)$ & $9(5 \%)$ & \\
\hline \multicolumn{4}{|l|}{ Blood pressure $(\mathrm{mm} \mathrm{Hg})^{\mathrm{a}}$} \\
\hline Systolic & $119.9 \pm 20.5$ & $119.7 \pm 19.0$ & 0.96 \\
\hline Diastolic & $76.3 \pm 9.6$ & $76.3 \pm 8.9$ & 0.97 \\
\hline Hypoxia & $10 / 40$ & $9 / 201$ & $<0.001$ \\
\hline Hypotension & $33 / 40$ & $31 / 201$ & $<0.001$ \\
\hline Hypertension & $11 / 40$ & $68 / 200$ & 0.43 \\
\hline Hypothermia & $2 / 40$ & $0 / 201$ & 0.03 \\
\hline Hyperthermia & $14 / 40$ & $50 / 200$ & 0.19 \\
\hline
\end{tabular}


Table 1 (Continued)

\begin{tabular}{|c|c|c|c|}
\hline Variable & Dead $(n=41)$ & Alive $(n=202)$ & $p$-Value \\
\hline \multicolumn{4}{|l|}{ CT characteristics } \\
\hline Time to CT (hours since injury) & $\begin{array}{l}5.6 \pm 7.9 \\
(n=33)\end{array}$ & $\begin{array}{l}8.0 \pm 15.8 \\
(n=184)\end{array}$ & 0.41 \\
\hline CT class-Marshall classification & & & 0.41 \\
\hline Class II & $14(34 \%)$ & $90(44.5 \%)$ & \\
\hline Class III & $8(20 \%)$ & $24(12 \%)$ & \\
\hline Class IV & $1(2 \%)$ & $1(0.5 \%)$ & \\
\hline Class V & $15(37 \%)$ & $71(35 \%)$ & \\
\hline Class VI & $3(7 \%)$ & $16(8 \%)$ & \\
\hline Petechial hemorrhage & $8(20 \%)$ & $39(19 \%)$ & 0.98 \\
\hline Obliteration of third ventricle or basal cisterns & $18(44 \%)$ & $49(24 \%)$ & 0.01 \\
\hline Subarachnoid hemorrhage & $17(41 \%)$ & $67(33 \%)$ & 0.31 \\
\hline Midline shift & $26(63 \%)$ & $103(51 \%)$ & 0.15 \\
\hline Nonevacuated hematoma & $6(15 \%)$ & $12(6 \%)$ & 0.053 \\
\hline Evacuated hematoma & $34(83 \%)$ & $181(90 \%)$ & 0.22 \\
\hline Extradural hematoma & $13(32 \%)$ & $76(38 \%)$ & 0.47 \\
\hline Subdural hematoma & $21(51 \%)$ & 89 (44\%) & 0.40 \\
\hline Contusion & $28(68 \%)$ & $118(58 \%)$ & 0.24 \\
\hline \multicolumn{4}{|l|}{ Blood investigations } \\
\hline Hemoglobin $(\mathrm{g} / \mathrm{dL})^{\mathrm{a}}$ & $\begin{array}{l}12.5 \pm 2.2 \\
(n=32)\end{array}$ & $\begin{array}{l}12.5 \pm 2.6 \\
(n=186)\end{array}$ & 0.94 \\
\hline Blood glucose (mg/dL) ${ }^{\mathrm{a}}$ & $\begin{array}{l}182.5 \pm 71.5 \\
(n=29)\end{array}$ & $\begin{array}{l}178.1 \pm 86.6 \\
(n=171)\end{array}$ & 0.77 \\
\hline Sodium (mEq/L)a & $\begin{array}{l}139.9 \pm 5.2 \\
(n=28)\end{array}$ & $\begin{array}{l}138.5 \pm 6.9 \\
(n=172)\end{array}$ & 0.20 \\
\hline Platelets $\left(\mathrm{mm}^{3}\right)^{\mathrm{a}}$ & $\begin{array}{l}200,560 \pm 80,926 \\
(n=25)\end{array}$ & $\begin{array}{l}244,784 \pm 97,526 \\
(n=162)\end{array}$ & 0.02 \\
\hline \multicolumn{4}{|l|}{ Other variables } \\
\hline Intubation at admission & $25(61 \%)$ & $72(36 \%)$ & 0.003 \\
\hline Surgery done & $34(83 \%)$ & $182(90 \%)$ & 0.18 \\
\hline Duration of hospital stay (d) ${ }^{\mathrm{a}}$ & $6.8 \pm 5.3$ & $8.8 \pm 7.9$ & 0.12 \\
\hline
\end{tabular}

Abbreviations: CT, computed tomography; ENT, ear nose throat; GCS, Glasgow coma scale.

aMean \pm standard deviations given for these variables; independent $t$-test used for comparison between groups.

Note: For all other variables, frequencies and percentages are given; Chi-square test or Fisher's exact test used for comparison between groups.

Significant $p$-values are shown in bold.

Table 2 Multivariable analysis

\begin{tabular}{|l|l|l|l|l|}
\hline \multirow{2}{*}{ Variables } & \multirow{2}{*}{ Odds ratio } & \multicolumn{2}{|l|}{ 95\% Cl for Exp (B) } & \multirow{2}{*}{$\boldsymbol{p}$-Value } \\
\cline { 3 - 6 } & & Lower & Upper \\
\hline GCS at admission & 0.845 & 0.730 & 0.979 & 0.025 \\
\hline Hypoxia & 8.164 & 2.187 & 30.481 & 0.002 \\
\hline Hypotension & 21.680 & 8.293 & 56.681 & $<0.001$ \\
\hline Obliteration of third ventricle/basal cisterns & 2.745 & 1.085 & 6.943 & 0.033 \\
\hline
\end{tabular}

Abbreviations: Cl, confidence interval; GCS, Glasgow coma scale.

those who had hypotension. Also, the odds of death were almost three times more for patients with obliteration of the third ventricle/basal cistern. The predictive power of the model was evaluated by using a receiver operating curve
(ROC curve). The model had $91.4 \%$ predictive power to discriminate between survived and expired patients (-Fig. 1). This means that our model is excellent for predicting in-hospital mortality in patients with moderate and severe 


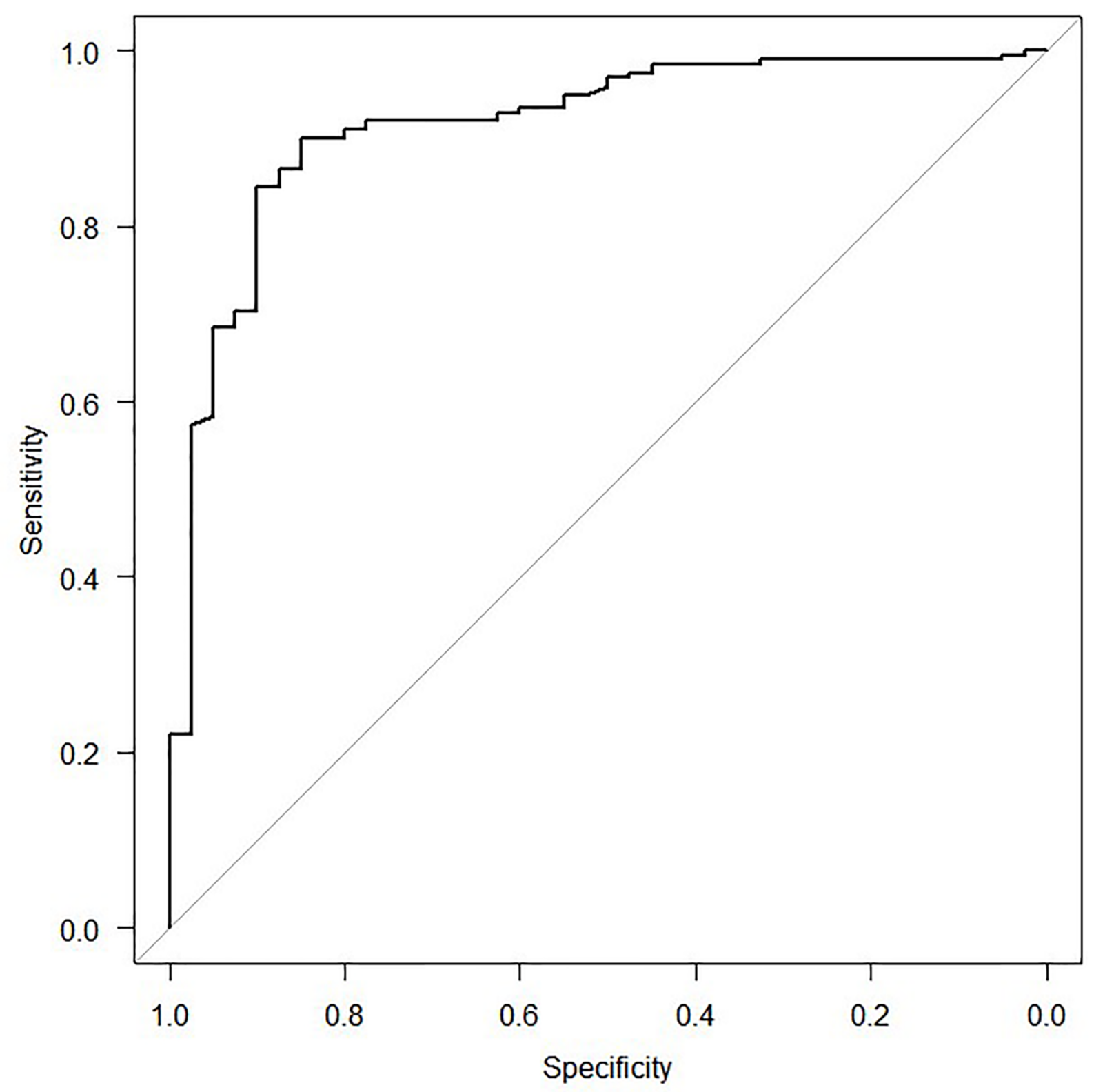

Fig. 1 The AUC for prognostic model iterations of mortality outcome, AUC: 0.914 (95\% confidence interval: 0.877-0.973). A value of AUC $>0.9$ indicates that the discriminatory ability of this model to predict in-hospital mortality is excellent. AUC, area under the receiver operating characteristic curve.

TBI treated in ICU. The TBI score obtained ranged from 0 to 4. A Chi-square test was performed to check the association between the score obtained and the survival of the patient, and the test was statistically significant (Chi-square $=85.38$, $\mathrm{df}=4, p$-value $<0.001$; - Table 3 ). The TBI score obtained from - Table 3 was rounded off to the nearest $5 \%$, and a simple score for predicting mortality is presented in - Table 4 .

\section{Traumatic Brain Injury Score}

Traumatic brain injury score was obtained based on the significant variables in multivariate logistic regression, that is, GCS at admission, hypoxia, hypotension, and obliteration third ventricle/basal cistern. The score was obtained by adding the individual scores of the above variables as given below.

$$
\begin{gathered}
\text { GCS at admission: } 3-4=+2,5-12=+1 \\
\text { Hypoxia: No }=0, \text { Yes }=+1 \\
\text { Hypotension: No }=0 \text {, Yes }=+1
\end{gathered}
$$

Obliteration third ventricle/basal cistern: $\mathrm{No}=0$, Yes $=+1$

The score values can range from 0 to 5 (when all the factors take the lowest level, that is, 0 , the total becomes zero and when all the factor levels are highest, it becomes $2+1+$ $1+1=5$ ) But in our data the maximum score obtained was 
Table 3 Traumatic brain injury score development

\begin{tabular}{|l|l|l|l|}
\hline \multirow{2}{*}{ TBI score } & Outcome & \multirow{2}{*}{ Total } \\
\cline { 2 - 3 } & Expired & Survived & 20 \\
\hline 0 & 0 & 20 & $100.0 \%$ \\
\hline \% within TBI score & $0.0 \%$ & $100.0 \%$ & 105 \\
\hline 1 & 4 & 101 & $100.0 \%$ \\
\hline \% within TBI score & $3.8 \%$ & $96.2 \%$ & 70 \\
\hline 2 & 8 & 62 & $100.0 \%$ \\
\hline$\%$ within TBI score & $11.4 \%$ & $88.6 \%$ & 40 \\
\hline 3 & 23 & 17 & $100.0 \%$ \\
\hline$\%$ within TBI score & $57.5 \%$ & $42.5 \%$ & 6 \\
\hline 4 & 5 & 1 & $100.0 \%$ \\
\hline \% within TBI score & $83.3 \%$ & $16.7 \%$ & \\
\hline
\end{tabular}

Abbreviation: TBI, traumatic brain injury.

Table 4 Simplified traumatic brain injury score

\begin{tabular}{|l|l|}
\hline TBI score & Mortality \\
\hline 0 & $0 \%$ \\
\hline 1 & $5 \%$ \\
\hline 2 & $10 \%$ \\
\hline 3 & $60 \%$ \\
\hline 4 & $85 \%$ \\
\hline
\end{tabular}

Abbreviation: TBI, traumatic brain injury.

Note: GCS at admission: $3-4=+2,5-12=+1$; hypoxia: no $=0$, yes $=+1$; hypotension: $n o=0$, yes $=+1$; obliteration third ventricle/ basal cistern: no $=0$, yes $=+1$.

only 4 , or in other words, all the factors considered here for scoring did not take the highest value simultaneously or otherwise, at least one of them took a lower level.

\section{Discussion}

We developed a prognostic model to predict in-hospital mortality based on variables at the time of admission in ICU. We used logistic regression to construct the model. The logistic regression has advantages over the other techniques, as it does not require variables to be normally distributed, linearly related, or to have equal within-group variances. Furthermore, logistic regression handles both categorical variables and continuous variables and gives us quickly interpretable outputs in the form of regression coefficients and odds ratios. ${ }^{1}$ We also used discrimination to assess the performance of the model. Our prognostic model discriminated excellently between patients who died from those who survived. Except for GCS and obliteration of the third ventricle/ basal cisterns, no significant association of other variables of the CRASH model with in-hospital mortality was found. Hypoxia and hypotension were also found as the most significant variables associated with death, which were also found in the IMPACT model.

The CRASH prognostic model is the result of the Medical Research Council CRASH meta-trial investigating the role of corticosteroids in patients with TBI. The CRASH model was developed from data of 10,008 patients with TBI enrolled from 1994 to 2004. ${ }^{13}$ The CRASH model is a popular prognostic model and is available as a web-based calculator. ${ }^{11}$ The CRASH model has been externally validated by the IMPACT database..$^{10}$ In the IMPACT validation study, discrimination of CRASH was lower than in the original data (C statistic 0.77 for both basic and CT models). The calibration was excellent for the CT model but poorer for the basic model..$^{13}$ The CRASH model has also been externally validated in various databases from developed countries..$^{9,10,14,15}$ Though the CRASH database included patients from low- to middle-income countries, it has not been externally validated in the database from these countries. The difference between the CRASH model and our model was different patient populations. We included patients with moderate and severe TBI who were admitted to ICU. This means that our patients had a more severe injury than most of the patients included in the CRASH database. We did not include patients who did not require intensive care, whereas the CRASH database included patients with all clinical severity (GCS = 3-14) irrespective of the place they were treated. The extracranial injury was shown to have a bearing on mortality in patients with TBI in the CRASH model. As our patient population was from neurosurgical ICU and we do not admit patients with polytrauma in this ICU, we did not have many patients with an extracranial injury. We included secondary complications like hypoxia and hypotension, which was not included in the CRASH model. The IMPACT model includes hypotension and hypoxia as variables. These are the significant contributor to mortality and morbidity in patients with severe TBI. ${ }^{16,17}$ Prognostication in TBI has substantially advanced in recent years. The IMPACT and CRASH models are mature, highly validated, and derived from vast datasets. In this context, the contribution of our prognostic model has demonstrated utility in a resource-poor environment. The goal of the IMPACT and CRASH model has been to prognosticate based on information available within the first 24 hours. However, the ability to prognosticate improves dramatically as the time from injury progresses.

An attempt has been made by others to predict outcomes in patients with TBI treated in ICU using commonly 
employed general ICU scales like APACHE II (Acute Physiology and Chronic Health Evaluation II), SAPS II (Simplified Acute Physiology Score II), and SOFA (Sequential Organ Failure Assessment). However, the simple prognostic model based only on age and GCS showed good prognostic performance, and the use of more complex general ICU scoring systems added little to this. ${ }^{18}$ However, the predictive power of the combined IMPACT-APACHE II models was found significantly superior compared with the original IMPACT model (AUC = $0.84-0.85$ vs. $0.81-0.82 ; p<0.05$ ) for 6 -month mortality prediction. ${ }^{19}$ This study indicates that besides admission variables, the addition of other ICU variables is useful in improving the accuracy of a standard prognostic model of TBI. Even in our present study, we found that the inclusion of secondary complications during ICU stays resulted in the superior performance of our model.

The performance of a prediction model is usually worse in external validation data than in the development data, and all prognostic models become outdated over time. The model may be customized to improve its performance and make it applicable to individual settings. Customization aims to enhance the performance of a particular prognostic model in a plausibly related but different population from the original development population. In general, customization does not affect discrimination but instead improves calibration ${ }^{20}$

\section{Limitations of the Study}

This was a retrospective cohort study with a small sample size. However, the research was done systematically, and the model construction and performance assessment were done by using standard methods like logistic regression and discrimination. ${ }^{1}$ We did not take 2 weeks of mortality as an endpoint for prediction; instead, we looked for in-hospital mortality. As the average time to death was $7.2 \pm 4.6$ days, well within 2 weeks, this endpoint is valid. The fact that the prognostic model works well in the hospital from which it is derived resulted in a perfect fit for our model. However, the generalizability of our model will require external validation in other setups.

\section{Conclusion}

We developed a new prognostic model for in-hospital mortality prediction in patients with moderate and severe TBI patients treated in our neurosurgical ICU. The performance of the model developed from our patient cohort was good. However, this model is not generalizable. It will require internal and external validation before applying it in any other setup. Future work is needed to establish the accuracy of our model in prospectively collected data of patients with TBI, both from our hospital and other hospitals in developing countries. Prediction models derived from datasets of the developed country are not generalizable to developing countries, and each hospital should have its prognostic model, which should be updated regularly.

\section{Note}

This study did not receive Institute Ethics Committee approval as it is mere a retrospective collection and analysis of data.

\section{Authors' Contributions}

D.S. and B.I.D. executed the design of study. M.J. and D.S. supported in data collection and data analysis. D.S., B.I.D., and D.I.B. carried out manuscript drafting.

\section{Funding}

B.I.D. is a member of the National Institute of Health Research Global Health Research Group on Neurotrauma. The latter is supported by the National Institute of Health Research using Official Development Assistance (ODA) funding (16/137/105).

\section{Conflict of Interest}

None declared.

\section{Acknowledgments}

The authors are grateful to Nikhil Ratna for the tabulation of results. The views expressed in this publication are those of the author(s) and not necessarily those of the NHS, National Institute for Health Research or the Department of Health.

\section{References}

1 Mushkudiani NA, Hukkelhoven CWPM, Hernández AV, et al. A systematic review finds methodological improvements necessary for prognostic models in determining traumatic brain injury outcomes. J Clin Epidemiol 2008;61(4):331-343

2 Maas AIR, Lingsma HF; IMPACT Study Group. New approaches to increase statistical power in TBI trials: insights from the IMPACT study. Acta Neurochir Suppl(Wien) 2008;101:119-124

3 Roozenbeek B, Maas AIR, Lingsma HF, et al; IMPACT Study Group. Baseline characteristics and statistical power in randomized controlled trials: selection, prognostic targeting, or covariate adjustment? Crit Care Med 2009;37(10):2683-2690

4 Perel P, Edwards P, Wentz R, Roberts I. Systematic review of prognostic models in traumatic brain injury. BMC Med Inform Decis Mak 2006;6:38

5 Menon DK, Zahed C. Prediction of outcome in severe traumatic brain injury. Curr Opin Crit Care 2009;15(5):437-441

6 Hukkelhoven CWPM, Rampen AJJ, Maas AIR, et al. Some prognostic models for traumatic brain injury were not valid. J Clin Epidemiol 2006;59(2):132-143

7 Maas AIR, Marmarou A, Murray GD, Teasdale SGM, Steyerberg EW. Prognosis and clinical trial design in traumatic brain injury: the IMPACT study. J Neurotrauma 2007;24(2):232-238

8 Roberts I, Yates D, Sandercock P, et al; CRASH trial collaborators. Effect of intravenous corticosteroids on death within 14 days in 10008 adults with clinically significant head injury (MRC CRASH trial): randomised placebo-controlled trial. Lancet 2004;364(9442):1321-1328

9 Raj R, Siironen J, Kivisaari R, et al. External validation of the international mission for prognosis and analysis of clinical trials model and the role of markers of coagulation. Neurosurgery 2013;73(2):305-311, discussion 311 
10 IMPACT. International mission for prognosis and analysis of clinical trials in TBI. Accessed September 4, 2014 at http:// www.tbi-impact.org/

11 CRASH. Clinical randomisation of an antifibrinolytic in significant haemorrhage prognostic model for predicting outcome after traumatic brain injury [Internet]. Accessed September 4, 2014 at http://www.crash2.lshtm.ac.uk/Risk calculator/index. html.

12 Dean AG, Sullivan KM, Soe MM. OpenEpi: open source epidemiologic statistics for public health [Internet]. [cited 2011 Jan 1]. Available at: www.OpenEpi.com. Accessed 2008

13 Perel P, Arango M, Clayton T, et al; MRC CRASH Trial Collaborators. Predicting outcome after traumatic brain injury: practical prognostic models based on large cohort of international patients. BMJ 2008;336(7641):425-429

14 Han J, King NKK, Neilson SJ, Gandhi MP, Ng I. External validation of the CRASH and IMPACT prognostic models in severe traumatic brain injury. J Neurotrauma 2014;31(13):1146-1152

15 Roozenbeek B, Lingsma HF, Lecky FE, et al; International Mission on Prognosis Analysis of Clinical Trials in Traumatic Brain Injury (IMPACT) Study Group; Corticosteroid Randomisation After Significant Head Injury (CRASH) Trial Collaborators; Trauma Audit and Research Network (TARN). Prediction of outcome after moderate and severe traumatic brain injury: external validation of the International Mission on Prognosis and Analysis of Clinical Trials (IMPACT) and Corticoid Randomisation After Significant Head injury (CRASH) prognostic models. Crit Care Med 2012;40(5):1609-1617

16 McHugh GS, Engel DC, Butcher I, et al. Prognostic value of secondary insults in traumatic brain injury: results from the IMPACT study. J Neurotrauma 2007;24(2):287-293

17 NoAuthorsListed. Hypotension. J Neurotrauma 2000;17(6-7): 591-595

18 Raj R, Skrifvars M, Bendel S, et al. Predicting six-month mortality of patients with traumatic brain injury: usefulness of common intensive care severity scores. Crit Care 2014;18(2):R60

19 Raj R, Siironen J, Kivisaari R, Hernesniemi J, Skrifvars MB. Predicting outcome after traumatic brain injury: development of prognostic scores based on the IMPACT and the APACHE II. J Neurotrauma 2014;31(20):1721-1732

20 Bakhshi-Raiez F, Peek N, Bosman RJ, de Jonge E, de Keizer NF. The impact of different prognostic models and their customization on institutional comparison of intensive care units. Crit Care Med 2007;35(11):2553-2560 